\title{
Can We Use Targeted Next-Generation Sequencing an Alternative Method to the Conventional Tests in Haematological Malignancies?
}

Emine Ikbal Atli ( $\nabla$ emine.ikbal@gmail.com )

Trakya Universitesi Tip Fakultesi https://orcid.org/0000-0001-9003-1449

Hakan Gurkan

Trakya Universitesi Tip Fakultesi

Engin Atli

Trakya Universitesi Tip Fakultesi

Hakki Onur Kirkizlar

Trakya Universitesi Tip Fakultesi

\section{Sinem Yalcintepe}

Trakya Universitesi Tip Fakultesi

Selma Demir

Trakya Universitesi Tip Fakultesi

Ufuk Demirci

Trakya Universitesi Tip Fakultesi

\section{Damla Eker}

Trakya Universitesi Tip Fakultesi

\section{Cisem Mail}

Trakya Universitesi Tip Fakultesi

\section{Rasime Kalkan}

Near East University

Ahmet Muzaffer Demir

Trakya Universitesi Tip Fakultesi

\section{Research}

Keywords: Myeloid malignancies, Haematological Malignancies, conventional tests, next generation sequencing (NGS)

Posted Date: August 17th, 2020

DOl: https://doi.org/10.21203/rs.3.rs-51331/v1 
License: (c) (i) This work is licensed under a Creative Commons Attribution 4.0 International License. Read Full License 


\section{Abstract}

Introduction: Advanced diagnostic methods give a huge advantage for identification of the abnormalities in myeloid malignancies. Researchers tried to show the potential importance of genetic tests both before the onset of the disease and during the remission. Large testing panels prevents false negative results in myeloid malignancies. But the important question is how can be merged with conventional cytogenetic and molecular cytogenetic techniques together with NGS technologies.

Methods: In this paper, we draw an algorithm for evaluation of the malignancies. In order to evaluation of genetic abnormities we performed cytogenetics, molecular cytogenetics and NGS testing panels in hematologic malignancies. In this study, we analyzed 132 patients which are referred to Medical Genetics Laboratory within different type of hematologic malignancies. We highlighted possible algorithm for cytogenetically normal cases.

Results: We analyzed cytogenetically normal patients by using NGS 141 gene panel and we detected two or more pathogenic variations in 20 out of 132 patients.

Conclusions: Despite of long turnaround time conventional techniques is still golden standard for myeloid malignancies but sometimes cryptic gene fusions or complex abnormalities cannot easily be identified by conventional techniques that conditions advanced technologies are recommended.

\section{Introduction}

Myeloid malignancies are originating from a hematopoietic progenitor cells and characterized by defective differentiation of myeloid progenitor cells [1]. Advanced molecular diagnostic techniques have been change the diagnostic algorithm. Increased usage of next generation sequencing (NGS) can help to change the scope, timing and suitability of genetic testing in hematologic malignancies [2]. Despite the increased advances in NGS technology and rising of study findings which supports the diagnostic and prognostic usage of mutational profiling in myeloproliferative neoplasms (MPN), the clinical decisionmaking role is still not fully utilized [3].

Diagnostics algorithms of acute myeloid leukemia (AML), myelodysplastic syndromes (MDS) and myeloproliferative neoplasms (MPN) has been changed in recent years [4,5]. Due to the advances in NGS technology various myeloid NGS panels are commercially available and generally analyse 25-50 genes which can be classified into several functional categories including the splicing machinery, epigenetic modifiers, transcription factors, signalling molecules and chromatin modifiers $[6,7,8,9]$.

The increased knowledge of genetic abnormalities has been updated classification of the Acute Myeloid Leukaemia (AML). The World Health Organization [11] and European LeukemiaNet [12] added new subgroups of diseases and molecular genetic abnormalities have also been added in diagnostic criteria's. Increased number of mutational, epigenetic and expression studies will be help identification of the novel markers in myeloid malignancies. 
National Comprehensive Cancer Network (NCCN) has been added mutations in FLT3, NPM1, CEBPA and KIT genes for risk evaluation [13] and according to the ELN guidelines TP53, RUNX1 and ASXL1 mutations should also be considered during the evaluation of risk. According to the studies; SF3B1, IDH1 and $I D H 2$ have also be added as a marker $[3,6,7,14,15]$. Laboratories should consider the risks for using large panels to test for myeloid malignancies. There are significant differences in intensity and variant outputs between different laboratories. Therefore, there is an urgent need to develop some standard diagnostic kits. In addition, existing somatic variant classification rules do not include complex interactions between variants within gene pathways [3]. Using NGS based myeloid gene panels will help for identification of multiple recurrent somatic mutations in many AML patients, and additional molecular genetic mutations can be detected in most cases, even within defined AML entities [2]. In myelodysplastic syndrome (MDS), NGS allows the detection of molecular mutations in approximately $90 \%$ of patients [6, $16,17]$.

As a result, the data of NGS should be interpreted in the context of other laboratory findings regarding cytomorphology, histopathology, immunophenotyping, conventional molecular genetics, cytogenetics, and clinical diagnostic parameters.

In this study, we analysed 149 patients which are referred to Medical Genetics Laboratory within different type of hematologic malignancies. We performed conventional cytogenetics, molecular cytogenetics and NGS analysis in these cases. According to the our results we highlighted possible algorithm for cytogenetically normal cases.

\section{Methods}

\section{Patient samples}

The present study included 132 patients (77 were men and 55 were women) from December 2017 to September 2018. Written informed consent was obtained from all cases. If patients are under 18 [9 children ( $\leq 15$ years)], from a parent and/or legal guardian were obtained. Study was approved by the Ethics Committee of our university and conducted in accordance with the ethical principles established in the Declaration of Helsinki. The median age of cases was 56 years, ranging from 1 to 90 years and there were 8 children ( $\leq 15$ years) in 132 adults. Patient characteristics were shown in Table 4 . Our cohort consists of 132 patients whose diagnosed with MM (39), AML (24), CLL (14), MDS (13), Lymphoma subtypes (13), ALL (6), Pancytopenia (4), MPD (2), Castleman Disease (2), Acute leucosis (2) and 13 other hematologic malignancies. DNA was isolated from bone marrow (QIAamp DNA Blood Mini Kit (bone marrow = 132) (Qiagen, Germany) and peripheral blood (MagNA Pure system Roche Diagnostics). DNA was quantified using a Qubit fluorometer (Thermo Fisher Scientific).

\section{Next generation sequencing}


In order to evaluation of myeloid neoplasm specific 141 genes The Human Myeloid Neoplasms QIAseq Targeted DNA Panel (Qiagen, Germany) was used. This panel covers exon/intron boundaries as shown in Figure 1 and covers genes has been listed oin Table 1 [18]. MiSeq sequencing-by synthesis bench top sequencer used for sequencing of amplified targets according to the manufacturer's protocol for pairedend sequencing (Illumina, San Diego, CA, USA). Data analysis and quality assessment for calling of single-nucleotide variants and analysis of short insertions and deletions evaluated by using Ingenuity Variant Analysis (IVA) programme. Amplicons were noted as a dropout and excluded from analysis if the coverage at any analysed position in any of the two paired-end sequences (minimal coverage) was 100x, with allele frequency $>5 \%$ were included for subsequent investigation. Known hotspot or clinically actionable variants detected below these thresholds were verified using orthogonal methods such as Sanger sequencing.

\section{Cytogenetic Assessment}

Karyotyping: Marrow Max and Chang media were used for cultures for bone marrow and peripheral blood specimens in a CO 2 incubator. After 24,48 , or $72 \mathrm{~h}$ of incubation culteres were harvested. Colcemid was used for arresting of metaphase cells and chromosome slides were stained by using for $\mathrm{G}$ banding protocol. International System for Human Cytogenetic Nomenclature (ISCN 2016) [19] was used for reporting and a least 20 metaphases were analysed in each culture.

Fluorescence in situ hybridization (FISH): FISH was applied according to the manufacturer's recommendations. A total of 200 interphase cells were analysed for each sample and images captured/stored by using Applied Imaging/Cytovision system. Final results were reported using the cutoff established in the laboratory for each of the probes tested [20]. Specific gene panels for FISH was applied for each malignancy. FISH panels for each of the malignancies are listed in below.

FISH Panel for MM: IGH/CCND1 t(11;14), CKS1B (1q21), IGH (14q32), p53 (17p13.1), 11q13/15q22/9q34, IGH/FGFR3 $t(4 ; 14)$, IGH/MAF t $(14 ; 16)$

FISH Panel for AML: 5q-, -5 (5p15, 5q31, 5q33), 7q-, -7 (Cen 7, 7q22, 7q31), Trisomy 8 (Cen 8), MLL (11q23), 20q- (20q12,20qter), RUNX1/RUNX1T1 (ETO/AML1) t(8;21), PML/RARA t(15;17), CBFB inv(16), $\mathrm{t}(16 ; 16)$

FISH Panel for KLL: 6q- (6q21), MYB (6q23), ATM (11q22.3), p53 (17p13.1), Trisomy 12 (Cen 12), 13q-/-13(13q14, 13q34), CCND1/lgH t(11;14)

FISH Panel for MDS: 5q-, -5 (5p15, 5q31, 5q33), 7q-, -7(Cen 7, 7q22, 7q31), Trisomy 8 (Cen 8), MLL(11q23), 20q- (20q12, 20qter)

FISH Panel for ALL: TCF3/PBX1 (E2A/PBX1) t(1;19), Trisomy or Tetrasomy 4, 6, 10, 17 (Cen 4, Cen 6, Cen 10, Cen 17), MYC(8q24), BCR/ABL1/ASS1 t(9;22), MLL (11q23), ETV6/RUNX1 (TEL/AML1) t(12;21), IgH (14q32) 
FISH Panel for Myeloproliferative Neoplasms (MPN): 5q, 7q, 8 Centromere/20q, BCR/ABL1/ASS t(9;22), FISH Panel for CML: Cen 8 (+ 8), BCR/ABL1-ASS1 t(9;22), RARA (17q21.2)/(iso17q)

\section{Results}

Characteristics of the 132 patients are summarized in Table 2. Among these patients, 77 were men and 55 were women. The median age was 56 years, ranging from 1 to 90 years and there were 124 adults and 8 children ( $\leq 15$ years). Cytogenetic and molecular cytogenetic evaluations were normal in all of the cases. By applying NGS using the 141 gene panel to the cytogenetically normal patients we found two or more pathogenic variations in 20 out of 132 patients. Detailed mutation list has been shown in Table 3.

\section{Next generation sequencing}

Next generation sequencing of hotspot regions of 141 gene has been performed in 132 bone marrow sample which are refereed from Department of Haematology. Variables with a depth of coverage $>100 x$ and an allele frequency of $>5 \%$ were included in this study. Known hot spots or variants identified below the threshold that may require clinically intervention were confirmed by using the Sanger sequencing. Variables of unknown significance were excluded from the clinical benefit analysis. Variants were classified as pathogenic and possible pathogenic according to the gene and clinical effects. Pathogenic variations were higher in patients with $\mathrm{AML}, \mathrm{MM}, \mathrm{CLL}$. Two or more pathogenic variations were identified in 20 out of 132 patients. List of the variants has been shown in Table 3. ASXL 1 c.1934dupg mutation was detected in AML and Myelofibrosis patients. NRAS c.181C > A was detected in $2 \mathrm{MM}$ patients, IDH2 c.419G > A was identified in AML and CMML cases. TET2 c.945delC mutation detected in AML and primary myelofibrosis cases, SRSF2 c.284C > A mutation was identified in AML and primary myelofibrosis cases. MPL c. 1544G > T mutation was detected in AML and primary myelofibrosis cases, SF3B1 c.2098A $>\mathrm{G}$ were identified in $\mathrm{AML}$, and MDS cases.

RUNX1 c.482T > C (AML, primary myelofibrosis) and U2AF1 c.101C > T (MDS) was classified as a possibly pathogenic. At the same time, 7 different variants of TP53 (TP53; c.568C >A, c.537T >A, c.596G $>A, c .503 A>G, c .818 G>A, c .395 A>G, c .638 G>T$ ) were detected in 7 different patients.

The lowest allelic frequency was observed in one MM patient in $\% 5, \% 20$ for pathogenic variation of KRAS: c. $183 \mathrm{~A}>\mathrm{C}$. These results show us clonally could be observed in lowest percentages. In the literature, it is recommended that to be able to determine clonally it should be evaluated up to $5 \%$ allelic fraction [21].

\section{Discussion}

Genetic and epigenetic alterations plays an important role during leukomogenesis [22]. Because of the limitations of the techniques, a variety of methods, from cytogenetic to complete genome sequencing, are needed to be able to prevent incomplete picture of genetic deviations in malignant homeopathies [23]. 
Advances in next generation sequencing (NGS) technology helps transformation of gene sequencing as a considerably faster and less expensive test, making it more practical in clinical practice. The validation of NGS panels are very critical and generally two-step approach is recommended for validation. The first one is related with optimization and analysis for relevant errors during the testing and the second step is related with establishment of thresholds of depth of coverage and VAF (low variant allele frequency of variations) for each type of identified variant [24].

Recent years, NGS has been used for identification of T cell clonality, recurrent cytogenetic translocations, identification of Philadelphia chromosome in Acute Lymphoblastic Leukaemia [2]. In addition of these conditions, in lymphoproliferative diseases NGS has been also used identification of clonal IGH and TCR rearrangements in MRD (Minimal Residual Disease) [21]. NGS technology can be used for identification of mutant or clonal DNA in several circulating tumor cells and with the increasing number of molecular markers, it is also essential for clinical trials based on substantially parallel next generation sequencing (NGS) [25].

Increased number of studies in this field will help to find out new mutations and gives chance to be updated WHO classification for myeloid malignancies. Moreover, those studies will be help for development of novel targeted therapeutic agents and novel therapeutic targets [26]. The discovery of new mutations in myeloid neoplasms enables us to understand the variable prognosis and pathogenesis of these diseases. The use of cytogenetic-based techniques allows the identification of "gross" chromosomal abnormalities such as translocations, amplifications, and deletions [22]. However the limitation of technique is based on size of the abnormality because genes can undergo changes in various ways (mutations, methylation, etc.) that may be critical for the onset and / or progression of malignant homeopathies. The major advance in NGS is identification of the molecular basis of leukemia because we can now classify malignant homeopathies at a molecular level that is more informative than the cytological classification [27].

Delic and colleagues analyzed 28-gene testing panel in different heamatologic malignencies (myeloproliferative neoplasms, essential thrombocythaemia, primary myelofibrosis, polycythaemia vera). Different mutations were identifed in splicing related genes (SF3B1, SRSF2 and U2AF1), chromatin modification genes (ASXL1 and EZH2) and methylation related genes (DNMT3A, IDH1, IDH2 and TET2) [28].

Here we performed sequencing of 141-gene on an Illumina MiSeq platform and highlighted its potential diagnostic importance for different haematological malignancies. We sequenced 141 genes in a cohort which consist of 132 patients whose diagnosed with MM (39), AML (24), CLL (14), MDS (13), Lymphoma subtypes (13), ALL (6), Pancytopenia (4), MPD (2), Castleman Disease (2), Acute leucosis (2) and 13 other hematologic malignancies.

We suggested to use this panel in routine clinical testing for myeloid malignancies which are cytogenetically and molecular cytogenetically reported as a normal. In this report, we identified variations in different genes which are related with epigenetic modifications, RNA modifications, transcription 
factors, DNA repair and cohesin complex. This shows us in patients which are cytogenetically or FISH were reported as a normal has to check in NGS panels. Thus, this will help to prevent false negative results in those cases and can help clinicians to be able to apply true treatment strategies. Our suggested possible algorithm was shown in Fig. 2. In this work, we analyzed pathogenic / possible pathogenic variations in different types of hematologic malignancies which were reported as a normal in cytogenetic and molecular cytogenetic analysis. This shows us only cytogenetic analysis does not sufficient for evaluation of diseases. In addition, NGS gives chance to analyze the genomic copy number alteration of interest gene which triggers different questions for conventional cytogenetics during the evaluation of myeloid neoplasms. The questions are; is it essential for both NGS-based tests and cytogenetics for evaluation of newly diagnosed myeloid neoplasm patients? Which technique is the best for monitoring patients during the treatment or during relapse?

To be able to answer these questions, we examined the similarities and differences between a conventional karyotype and a targeted NGS panel.

1- Conventional cytogenetic gives information for both numerical and structural abnormalities of chromosomes but NGS is target specific and can give only numerical abnormalities. We cannot used NGS for identification of insertion or translocations.

2- Karyotype produces a genomic information in single cell resolution, but NGS provides information for a "mean" copy number changes for all cells in a sample. Also, karyotype gives chance to be able to identification of the low percentage clones which contains different aberrations. On the other side, NGS panels identifies only numerical alterations which are shared with a significant portion of the cells in the sample (typically $>30 \%$ ).

3- Third, the resolution of karyotype analysis is usually > $10 \mathrm{Mb}$ while NGS panels offers resolution at the gene or exon level. At the same time, cytogenetic analysis cannot be identified submicroscopic abnormalities (10 Mb) but NGS panels can do.

4- If the aneuploidy is high, the ability of karyotype analysis becomes limited. For example, in the case of a complex karyotype which contains a small "marker chromosomes", it is difficult to identify the chromosomal origin of this abnormality. But targeted NGS panels identifies these abnormalities rapidly and reliably.

5- NGS panels measures copy numbers in relative and inaccurate proximity. The triploidy or tetraploid genome cannot be easily distinguished from the diploid genome. This can be easily determined by karyotype analysis.

These differences demonstrate that the advantages and disadvantages of targeted NGS and karyotype / targeted FISH techniques which supports the idea that complementary technologies are best used to evaluate the numerical and structural properties of neoplastic genomes. 
NGS-based panel testing is widely accepted in clinical practice, and this can facilitate the construction of well-designed comprehensive NGS panels, especially during initial diagnosis. Albeit, the targeted NGS panels can evaluate the genome wide numerical imbalances, it cannot identify structural abnormalities, lack of single cell resolution and low target density, necessitate simultaneous cytogenetic analysis capable of presenting a complete picture of the genomic profile. After the diagnostic evaluation, it may be gives advantage to use NGS panel testing to perform cytogenetic analysis for patients whose NGS results show significant clonal evolution.

In this study, we provided an information about the potential uses of NGS in a routine clinical setting and emphasized the importance of the method for clinical studies and improving outcomes of haematology patients. NGS gives chance to new perspective for personalized diagnostics and targeted therapy opportunity for myeloid malignancies. The advanced sensitivity and accuracy makes NGS as a potential diagnostic test in cytogenetically normal myeloid malignancies. Identification of novel specific mutations can be help to find out new targeted therapy options which would be a cornerstone of personalized medicine in hematologic malignancies. Finally, this study provides evidence on potential uses of NGS in the routine clinical setting, and provides a framework for cytogenetically normal cases.

\section{Declarations}

\section{Ethical approval}

All procedures performed in the study involving human participants were in accordance with the ethics committee of the Trakya University Faculty of Medicine and followed Declaration of Helsinki.

\section{Consent for publication}

Informed consent was obtained from all individual participants included in the study.

\section{Availability of data and material}

The datasets used or analyzed during the current study are available from the corresponding author on reasonable request.

\section{Competing Interests Statement}

The authors declare that they have no conflict of interest.

\section{Funding Statement}

The author(s) received no specific funding for this work.

\section{Authors' contributions}


E.I.A.- R.K. designed and performed experiments and wrote the manuscript, E.A. and D.E.-C.M. performed experiments, U.D. - A.M.D. and H.O.K. collected and characterised patients, E.I.A - H.G. and E.A. performed analysis, S.D. and S.Y. proofread and wrote parts of the manuscript. All authors read and approved the final version of the manuscript.

\section{Acknowledgements}

We would like to thank all of the participants in this study.

\section{References}

1. Visconte V, O Nakashima M, J Rogers H. Mutations in Splicing Factor Genes in Myeloid Malignancies: Significance and Impact on Clinical Features. Cancers (Basel). 2019 Nov 22;11(12). pii: E1844. doi: 10.3390/cancers11121844.

2. Palumbo GA et al. The Role of New Technologies in Myeloproliferative Neoplasms. Front Oncol. 2019 Apr 26;9:321. doi: 10.3389/fonc.2019.00321. eCollection 2019.

3. Bacher $U$ et al. Challenges in the introduction of next-generation sequencing (NGS) for diagnostics of myeloid malignancies into clinical routine use. Blood Cancer J. 2018 Nov 12;8(11):113. doi: 10.1038/s41408-018-0148-6.

4. Shumilov, E. et al. Current status and trends in the diagnostics of AML and MDS. Review article. Blood Rev. (2018). https://doi.org/10.1016/j.blre.04.008.

5. Barbui, T. et al. The 2016 WHO classification and diagnostic criteria for myeloproliferative neoplasms: document summary and in-depth discussion. Blood Cancer J. 8 (2018). https://doi.org/10.1038/s41408-018-0054-y

6. Cazzola, M., Della Porta, M. G. \& Malcovati, L. The genetic basis of myelodysplasia and its clinical relevance. Blood 122, 4021-4034 (2013).

7. Papaemmanuil, E. et al. Genomic classification and prognosis in acute myeloid leukemia. N. Engl. J. Med. 374, 2209-2221 (2016).

8. Papaemmanuil, E. et al. Clinical and biological implications of driver mutations in myelodysplastic syndromes. Blood 122, 3616-3627 (2013).

9. Haley J. Abel, Eric J. Duncavage, Detection of structural DNA variation from next generation sequencing data: a review of informatic approaches, Cancer Genetics, 206,432- 440, (2013) ISSN 2210-7762, https://doi.org/10.1016/j.cancergen.2013.11.002.

10. Duncavage, E. J. \& Tandon, B. The utility of next-generation sequencing in diagnosis and monitoring of acute myeloid leukemia and myelodysplastic syndromes. Int. J. Lab. Hematol. 37(Suppl 1), 115121 (2015).

11. Arber, D. A. et al. The2016 revision to the World Health Organization classification of myeloid neoplasms and acute leukemia. Blood 127, 2391-2405 (2016). 
12. Dohner H. et al. Diagnosis and management of AML in adults: $2017 \mathrm{ELN}$ recommendations from an international expert panel. Blood 129:424-447(2017)

13. Patel U, Luthra R, Medeiros LJ, Patel KP. Diagnostic, Prognostic, and Predictive Utility of Recurrent Somatic Mutations in Myeloid Neoplasms. Clin Lymphoma Myeloma Leuk. 2017 Jul;17S:S62-S74. doi: 10.1016/j.clml.2017.02.015.

14. Cancer Genome Atlas Research. Genomic and epigenomic landscapes of adult de novo acute myeloid leukemia. N. Engl. J. Med. 368, 2059-2074 (2013).

15. Duncavage, E. J. \& Tandon, B. The utility of next-generation sequencing in diagnosis and monitoring of acute myeloid leukemia and myelodysplastic syndromes. Int. J. Lab. Hematol. 37(Suppl 1), 115121 (2015).

16. Yoshida $\mathrm{K}$ et al. Frequent pathway mutations of splicing machinery in myelodysplasia. Nature 478 : 64-69(2011)

17. Bacher, U., Kohlmann, A. \& Haferlach, T. Mutational profiling in patients with MDS: ready for everyday use in the clinic? Best. Pract. Res. Clin. Haematol. 28,32-42 (2015)

18. A Sample to Insight ${ }^{\circledR} N G S$ solution for myeloid neoplasms: Redefined amplicon sequencing for low variant detection and interpretation (Application Note: PROM-12533- 001)(2018)

19. Howe, B., Umrigar, A., Tsien, F. Chromosome Preparation From Cultured Cells. J. Vis. Exp. (83), e50203, doi:10.3791/50203 (2014).

20. Rack, K.A. et al. European recommendations and quality assurance for cytogenomic analysis of haematological neoplasms. Leukemia 33, 1851-1867 (2019) doi:10.1038/s41375-019-0378-z

21. Gazzola A. et al. The evolution of clonality testing in the diagnosis and monitoring of hematological malignancies. Therapeutic Adv Hematol. (2014) 5:35- 47. doi:10.1177/2040620713519729

22. Etienne De Braekeleer, Nathalie Douet-Guilbert \& Marc De Braekeleer (2014) Genetic diagnosis in malignant hemopathies: from cytogenetics to next-generation sequencing, Expert Review of Molecular Diagnostics, 14:2, 127-129, DOI:10.1586/14737159.2014.872563

23. Mitelman F, Johansson B, Mertens F, editors. Mitelman Database of Chromosome Aberrations and Gene Fusions in Cancer. 2013. Available from: http://cgap.nci.nih.gov/Chromosomes/Mitelman

24. Kim H, Yun JW, Lee ST, Kim HJ, Kim SH, Kim JW; Korean Society for Genetic Diagnostics Clinical Guidelines Committee. Korean Society for Genetic Diagnostics Guidelines for Validation of NextGeneration Sequencing-Based Somatic Variant Detection in Hematologic Malignancies. Ann Lab Med. 2019 Nov;39(6):515-523. doi:10.3343/alm.2019.39.6.515.

25. Avila and Meric Bernstam. Next-Generation Sequencing for the General Cancer Patient. Clin Adv Hematol Oncol. 17(8):447-454(2019)

26. National Comprehensive Cancer Network. Myeloproliferative neoplasms (Version 2.2018). Available from: https://www.nccn.org/professsionals/physician_gls/pdf/mpn.pdf.Accessed September 7, 2017. 
27. Kuo FC, Steensma DP, Dal Cin P. Conventional cytogenetics for myeloid neoplasms in the era of next generation sequencing. AmJHematol. 2017;92:227229. https://doi.org/10.1002/ajh.24642

28. Delic S, Rose D, Kern W, Nadarajah N, Haferlach C, Haferlach T, Meggendorfer M. Application of an NGS-based 28-gene panel in myeloproliferative neoplasms reveals distinct mutation patterns in essential thrombocythaemia, primary myelofibrosis and polycythaemia vera. Br J Haematol. 2016 Nov;175(3):419-426. doi: 10.1111/bjh.14269. Epub 2016 Jul 22.

\section{Tables}


Table 1.

The list of covered genes and related diseases in NGS panel.

\section{Disease Genes covered}

\section{Acute} lymphoblastic leukaemia (ALL)

Acute myeloid leukaemia (AML)
ASXL2, ATM, BRAF, CALR, CDKN2A, CREBBP, CRLF2, CSF3R, CTCF, DNM2, EGFR, EP300, FBXW7, GATA2, HNRNPK, HRAS, IKZF3, IL 7R, KDM6A, KDR, KMT2C, LRRC4, MAP2K1, MLH1, MSH2, MSH6, NOTCH1, NTRK3, PAX5, PDGFRA, PMS2, PRAMEF2, PTEN, RELN, SMARCB1

ANKRD26, ASXL1, ATM, BCOR, BCORL 1, BIRC3, BRAF, C17orf97, CALR, CARD11, CBLC, CDKN2A, CEBPA, CHEK2, CREBBP, CSF1R, CSF3R, CTCF, DAXX, DDX41, DNM2, DNMT1, ELANE, EP300, FLRT2, FLT3, GATA1, GATA2, HNRNPK, IDH1, IDH2, IKZF1, IL 7R, JAK1, JAK3, KDM6A, KDR, KIT(CD117), KMT2A, KMT2C, KRAS, LRRC4, MAP2K1, MPL, MSH6, MYC, NBN, NOTCH1, NPM1, NRAS, NSD1, NTRK3, OR13H1, OR8B12, P2RY2, PCDHB1, PDGFRA, PHF6, PRAMEF2, PRPF8, PTEN, PTPN11, RAD21, RUNX1 (AML1), SF1, SF3A1, SMARCB1, SMC1A (SMC1L1), SMC3, SRP72, SRSF2, STAG2, STXBP2, U2AF1, U2AF2, WT1

Chronic lymphocytic leukaemia (CLL)

Chronic myeloid leukaemia (CML) ADA, BIRC3, BLM, BRAF, CALR, CHEK2, CSF3R, KCNA4, KLHL6, KMT2C, MAP2K1, NBN, NPAT, NTRK3, OR13H1, OR8B12, PRAMEF2, SRP72, TAL1, TERT, TUBA3C, WAS, WRN

ABL 1, CALR, CDKN2A, CEBPA, CREBBP, CSF1R, CSF3R, FBXW7, GATA2, KDM6A, $M S H 2, M S H 6, R B 1, S M C 1 A$ (SMC1L 1), TP53

Chronic myelomonocytic leukaemia (CMML)

Chronic neutrophilic leukaemia (CNL)

CALR, CEBPA, CSF1R, CSF3R, HRAS, KMT2C, LUC7L2, SRSF2
Multiple myeloma

Myelodysplastic syndromes (MDS)

Myeloid malignancies

Myeloproliferative neoplasm (MPN)

Myelofibrosis (MF)

Other myeloid neoplasms

Other myeloid neoplasm genes
ATM, BCL6, BCR, BIRC3, BRAF, CDKN2A, CEBPA, EGFR, FBXW7, GJB3, HRAS, KDM6A, MYC, NOTCH1, PTEN, SH2D1A, SMARCB1

ATRX, CALR, CDKN2A, CEBPA, CSF1R, CSF3R, EP300, ETNK1, GNAS, HRAS, KDM6A, KMT2A, KMT2C, RAD21, RB1, SETBP1, SF1, SF3A1, SMC3, SRSF2, STAG2, U2AF1, U2AF2, XPO1, ZRSR2

CBL, CBLB, DNMT3A, EED, ETV6, EZH2, PRPF4OB, SUZ12, TET2, TP53

ABL 1, ASXL1, CALR, CSF1R, JAK2, JAK3, KAT6A (MYST3), KRAS, MPL, NF1, NRAS, RB1, SETBP1, SF3B1, SH2B3, SRSF2, STAG2.

CALR, CHEK2, IDH1, IDH2, CSF1R, SRSF2

BRAF, CDKN2A, CEBPA, FBXW7, HRAS, IKZF3, KLHDC8B, KMT2C, MSH6, NTRK3, PTEN, SRP72, TPMT

BRCA1, BRCA2, BRINP3, CUX1, FAM47A, FAS, KCNK13, MYD88, PML, PRF1, SAXO2, STAT3, TERC, TNFRSF13B 
Table 2.

WHO classification of our cohort and results of genetic analysis.

\begin{tabular}{|c|c|c|c|c|c|c|}
\hline WHO classification & $\mathrm{n}$ & $\begin{array}{l}\text { Detected } \\
\text { pathogenic } \\
\text { variant }\end{array}$ & $\begin{array}{l}\text { Detected likely } \\
\text { pathogenic } \\
\text { variant }\end{array}$ & $\begin{array}{l}\text { Detected } \\
\text { Vous }\end{array}$ & FISH & Karyotype \\
\hline $\begin{array}{l}\text { Multiple } \\
\text { Myeloma }\end{array}$ & 39 & 15 & 2 & 12 & $\mathrm{~N}$ & $\mathrm{~N}$ \\
\hline $\begin{array}{l}\text { Acute myeloid } \\
\text { leukemia (AML) }\end{array}$ & 24 & 18 & 11 & 15 & $\mathrm{~N}$ & $\mathrm{~N}$ \\
\hline $\begin{array}{l}\text { Chronic lymphocytic } \\
\text { leukemia (KLL) }\end{array}$ & 14 & 16 & - & 16 & $\mathrm{~N}$ & $\mathrm{~N}$ \\
\hline $\begin{array}{l}\text { Myelodysplastic } \\
\text { syndrome (MDS) }\end{array}$ & 13 & 4 & - & 4 & $\mathrm{~N}$ & $\mathrm{~N}$ \\
\hline $\begin{array}{l}\text { Acute lymphocytic } \\
\text { leukemia (ALL) }\end{array}$ & 6 & 3 & 6 & 2 & $\mathrm{~N}$ & $\mathrm{~N}$ \\
\hline $\begin{array}{l}\text { Lymphoma and } \\
\text { subtypes }\end{array}$ & 13 & 3 & 1 & 7 & $\mathrm{~N}$ & $\mathrm{~N}$ \\
\hline Pancytopenia & 4 & - & - & 3 & $\mathrm{~N}$ & $\mathrm{~N}$ \\
\hline $\begin{array}{l}\text { Chronic } \\
\text { myeloproliferative } \\
\text { diseases (MPD) }\end{array}$ & 2 & 3 & - & - & $\mathrm{N}$ & $\mathrm{N}$ \\
\hline Castleman disease & 2 & - & - & 1 & $\mathrm{~N}$ & $\mathrm{~N}$ \\
\hline Acute leukosis & 2 & 2 & - & 1 & $\mathrm{~N}$ & $\mathrm{~N}$ \\
\hline Primary myelofibrosis & 1 & 4 & 1 & & $\mathrm{~N}$ & $\mathrm{~N}$ \\
\hline $\begin{array}{l}\text { Chronic } \\
\text { myelomonocytic } \\
\text { leukemia (CMML) }\end{array}$ & 1 & 1 & - & - & $\mathrm{N}$ & $\mathrm{N}$ \\
\hline Plasma cell leukemia & 1 & - & - & - & $\mathrm{N}$ & $\mathrm{N}$ \\
\hline Isolated neutropenia & 1 & 1 & - & - & $\mathrm{N}$ & $\mathrm{N}$ \\
\hline Bicytopenia & 1 & - & - & - & $\mathrm{N}$ & $\mathrm{N}$ \\
\hline $\begin{array}{l}\text { Essential } \\
\text { thrombocytosis }\end{array}$ & 1 & - & - & - & $\mathrm{N}$ & $\mathrm{N}$ \\
\hline Hemolytic anemia & 1 & - & - & - & $\mathrm{N}$ & $\mathrm{N}$ \\
\hline Others & 6 & 1 & 1 & 1 & $\mathrm{~N}$ & $\mathrm{~N}$ \\
\hline
\end{tabular}


Table 3. The list of identified mutations and their distributions of diseases.

Page 15/19 


\begin{tabular}{|c|c|}
\hline Mutation List & Disorders \\
\hline TP53 c.568C >A & LYMPHOMA \\
\hline TP53 с. $537 \mathrm{~T}>\mathrm{A}$ & AML \\
\hline TP53 с.596G >A & AML \\
\hline TP53 с. $503 \mathrm{~A}>\mathrm{G}$ & AML \\
\hline TP53 с.818G>A & $\mathrm{MM}$ \\
\hline TP53 с.395A>G & CLL \\
\hline TP53 c.638G >T & CLL \\
\hline NRAS c.35G $>$ A & LYMPHOMA \\
\hline \multirow[t]{2}{*}{ NRAS c.181C>A } & $\mathrm{MM}$ \\
\hline & $\mathrm{MM}$ \\
\hline NRAS c.183A>T & $\mathrm{MM}$ \\
\hline JAK2 с.1748G >A & LYMPHOMA \\
\hline JAK2 с.3G $>A$ & CASTLEMAN D. \\
\hline \multirow[t]{2}{*}{ IDH2 c.419G>A } & AML \\
\hline & CMML \\
\hline PTPN11 с.227A>G & AML \\
\hline PTPN11 c.184T>G & ACUTE LEUKOSIS \\
\hline PTPN11 c.205G>A & CLL \\
\hline TET2 с. $2746 \mathrm{C}>\mathrm{T}$ & $\mathrm{AML}$ \\
\hline \multirow[t]{2}{*}{ TET2 c.945delC } & AML \\
\hline & PRIMARY MYELOFIBROSIS \\
\hline TET2 c.3543_3544delCT & AML \\
\hline TET2 c. $4182+1 \mathrm{G}>\mathrm{A}$ & MPD \\
\hline TET2 c.822delC & ALL \\
\hline FLT3 c.1784_1804dupGAGAATATGAATATGATCTCA & AML \\
\hline FLT3c.1770_1793dupCTACGTTGATTTCAGAGAATATGA & AML \\
\hline FLT3 с. $2678 \mathrm{C}>\mathrm{T}$ & MM \\
\hline FLT3 c.1748G >A & CLL \\
\hline FLT3c.1810_1811insGGGAATATGAATATGATCTCAAATGGG & ALL \\
\hline FLT3c.1755_1778dupAGATAATGAGTACTTCTACGTTGA & AML \\
\hline SF3B1 c.1866G >T & $\mathrm{MM}$ \\
\hline SF3B1 c.1866G > T & CLL \\
\hline SF3B1 с.2225G>A & CLL \\
\hline NOTCH1 c. $4721 \mathrm{~T}>\mathrm{C}$ & ALL \\
\hline NOTCH1 c.7541_7542delCT & CLL \\
\hline NOTCH1 с.7330C >T & CLL \\
\hline BIRC3 c.1639delC & ISOLATED NEUTROPENIA \\
\hline WT1 c.1153_1157dupCGGTC & AML \\
\hline ASXL1 c.1141G $>\mathrm{T}$ & $\mathrm{MM}$ \\
\hline ASXL1 c.1900_1922delAGAGAGGCGGCCACCACTGCCAT & $\mathrm{MM}$ \\
\hline ASXL1 c.2129delG & MPD \\
\hline \multirow[t]{3}{*}{ ASXL1 c.1934dupG } & $\mathrm{AML}$ \\
\hline & $\mathrm{AML}$ \\
\hline & PRIMARY MYELOFIBROSIS \\
\hline ATM c. $7328 \mathrm{G}>\mathrm{A}$ & AML \\
\hline ATM c. $2250+2 \mathrm{~T}>\mathrm{A}$ & $\mathrm{MM}$ \\
\hline ATM c.6848_6855delCAGTTAGC & CLL \\
\hline CHEK2 c.1312G > T & ALL \\
\hline BCORL1 с.2916T>A & $\mathrm{AML}$ \\
\hline NF1 c. $4537 \mathrm{C}>\mathrm{T}$ & AML \\
\hline \multirow[t]{2}{*}{ SRSF2 c.284C $>$ A } & AML \\
\hline & PRIMARY MYELOFIBROSIS \\
\hline
\end{tabular}

Page 16/19 


\begin{tabular}{|c|c|}
\hline DNMT3A c.2645G>A & MM \\
\hline \multirow[t]{2}{*}{ MPL c.1544G > T } & AML \\
\hline & PRIMARY MYELOFIBROSIS \\
\hline NPM c.860_863dupTCTG & ACUTE LEUKOSIS \\
\hline TNFRSF13B c.310T>C & $\mathrm{MM}$ \\
\hline KRAS c.182A>G & MM \\
\hline KRAS c.183A>C & $\mathrm{MM}$ \\
\hline TERT с. $2035 \mathrm{~T}>\mathrm{G}$ & $\mathrm{MM}$ \\
\hline MYD88 c.684G>A & MM \\
\hline BRCA2 c.4446_4451dupAACAGA & MDS \\
\hline BRCA2 c.9097dupA & CLL \\
\hline NBN c. $871 C>T$ & CLL \\
\hline RB1 c.2431delA & CLL \\
\hline XPO1 c.1711G>C & CLL \\
\hline GATA2 c. $1076 \mathrm{~T}>\mathrm{C}$ & MPD \\
\hline PHF6 c.1024C>T & ALL \\
\hline KDM6A c.1578delG & BONE MARROW INVOLVEMENT \\
\hline EP300 c.6627_6638delCCAGTTCCAGCA & AML \\
\hline \multirow[t]{2}{*}{ SF3B1 с.2098A>G } & $\mathrm{AML}$ \\
\hline & MDS \\
\hline SF3B1 c.1866G > T & AML \\
\hline ASXL1 с.2056A>T & AML \\
\hline ATM c.7466C > T & CLL \\
\hline ATM c.3032delC & ALL \\
\hline CHEK2 c. $444+1 \mathrm{G}>\mathrm{A}$ & HODGKIN LENFOMA \\
\hline CHEK2 c.480A > G & SYSTEMIC MASTOCYTOSIS \\
\hline ETV6 c. $163+1 \mathrm{G}>\mathrm{T}$ & AML \\
\hline SRSF2 c. $284 \mathrm{C}>\mathrm{T}$ & ALL \\
\hline \multirow[t]{2}{*}{ RUNX1 с.482T>C } & $\mathrm{AML}$ \\
\hline & PRIMARY MYELOFIBROSIS \\
\hline DNMT3A с. $2645 \mathrm{G}>\mathrm{A}$ & $\mathrm{AML}$ \\
\hline DNMT3A c.976delC & NHL \\
\hline CREBBP c.5213_5216dupATGC & $\mathrm{AML}$ \\
\hline STAG2 c.1414G $>$ T & AML \\
\hline CEBPA c.779_783delACCCCinsG & AML \\
\hline GNAS c. $1376 C>$ G & $\mathrm{MM}$ \\
\hline BCOR c. $2428 \mathrm{C}>\mathrm{T}$ & MDS \\
\hline BCORL1 с.2916T>A & AML \\
\hline IKZF3 c.485T>G & CLL \\
\hline CBLB c. $2008 \mathrm{C}>\mathrm{T}$ & CLL \\
\hline \multirow[t]{2}{*}{ U2AF1 с.101C>T } & MDS \\
\hline & MDS \\
\hline FBXW7 c.1393C > T & ALL \\
\hline ASXL1 c.143G > T & $\mathrm{MM}$ \\
\hline \multirow[t]{2}{*}{ SF3B1 c. $422 A>G$} & $\mathrm{MM}$ \\
\hline & MM \\
\hline NOTCH1 c.7357delG & CLL \\
\hline ATM c.7466C $>\mathrm{T}$ & AML \\
\hline ATM c.3605G >C & MDS \\
\hline BCORL1 с. $2446 \mathrm{C}>\mathrm{T}$ & CLL \\
\hline NF1 c.1921A>G & MANTLE CELL LENFOMA \\
\hline ETV6 c.909dupC & DBBHL \\
\hline \multirow[t]{2}{*}{ MPL c.121T >C } & $\mathrm{AML}$ \\
\hline & CLL \\
\hline MPL с.1481T>G & $\mathrm{MM}$ \\
\hline DNMT3A c.1555-8_15551delCTGTCTAG & $\mathrm{AML}$ \\
\hline MYD88 c.815G>A & MM \\
\hline
\end{tabular}




\begin{tabular}{|c|c|}
\hline BCORL1 c. $2446 \mathrm{C}>\mathrm{T}$ & CLL \\
\hline NBN c.163_171+3delACCAACCTGGTA & CLL \\
\hline IKZF3 c.998A>G & CLL \\
\hline CBLB c. $2434 \mathrm{G}>\mathrm{A}$ & $\mathrm{MM}$ \\
\hline CBLB c.1927A>G & $\mathrm{MM}$ \\
\hline CBLB c. $1472 A>G$ & $\mathrm{MM}$ \\
\hline CBLB c. $815 G>A$ & MDS \\
\hline JAK1 c. $2948 \mathrm{~A}>\mathrm{G}$ & BONE MARROW INVOLVEMENT \\
\hline JAK1 c. $1951 \mathrm{G}>\mathrm{A}$ & AML \\
\hline JAK1 с.2221G >A & CLL \\
\hline DNM2 c. $-4 \mathrm{C}>\mathrm{T}$ & FOLLICULAR LYMPHOMA \\
\hline EP300 с.7238T>A & PANCYTOPENIA \\
\hline EP300 с.6967C >T & CLL \\
\hline BLM c.3416G >C & MANTLE CELL LENFOMA \\
\hline \multirow[t]{2}{*}{ BLM c. $274 \mathrm{~A}>\mathrm{G}$} & LYMPHOMA \\
\hline & ALL \\
\hline BLM c. $2237 \mathrm{C}>\mathrm{T}$ & $\mathrm{MM}$ \\
\hline BLM c.1693G >A & CLL \\
\hline EZH2 с.943A>G & DBBHL \\
\hline AKAP13 c.7265G>A & AML \\
\hline DNAH9 с.10555C >A & AML \\
\hline SMC1A c. $2152 \mathrm{G}>\mathrm{A}$ & AML \\
\hline OR8B12 c.353C > T & AML \\
\hline CALR c. $-2 \mathrm{C}>\mathrm{T}$ & CLL \\
\hline \multirow[t]{3}{*}{ CALR c.682C $>$ T } & $\mathrm{AML}$ \\
\hline & MDS \\
\hline & CLL \\
\hline KAT6A c.4108G >A & AML \\
\hline ASXL2 с.833T >A & AML \\
\hline NPM1 c.733G>C & AML \\
\hline PRPF40B c.1103C >T & $\mathrm{AML}$ \\
\hline PMS2 с.2321A >T & AML \\
\hline $\mathrm{ADA}$ с. $179 \mathrm{~A}>\mathrm{G}$ & AML \\
\hline \multirow[t]{2}{*}{ SETD2 c.6685G>A } & AML \\
\hline & CLL \\
\hline PAX5 с.1096C>T & ACUTE LEUKOSIS \\
\hline AKAP13 с. $7265 \mathrm{G}>\mathrm{A}$ & $\mathrm{MM}$ \\
\hline CSF3R c.355G >A & $\mathrm{MM}$ \\
\hline SF3A1 c.458T>C & $\mathrm{MM}$ \\
\hline IKZF1 c.949_951delAAC & PANCYTOPENIA \\
\hline СNOT3 c.1847C > T & PANCYTOPENIA \\
\hline NTRK3 c. $121 \mathrm{~A}>\mathrm{G}$ & MDS \\
\hline NTRK3 с.2293-3C > T & CLL \\
\hline ZRSR2 c.1098_1099delGA & CLL \\
\hline ETNK1 с. $749 \mathrm{C}>\mathrm{T}$ & CLL \\
\hline IL7R c.602A>G & CLL \\
\hline TUBA3C c.544G >A & CLL \\
\hline
\end{tabular}




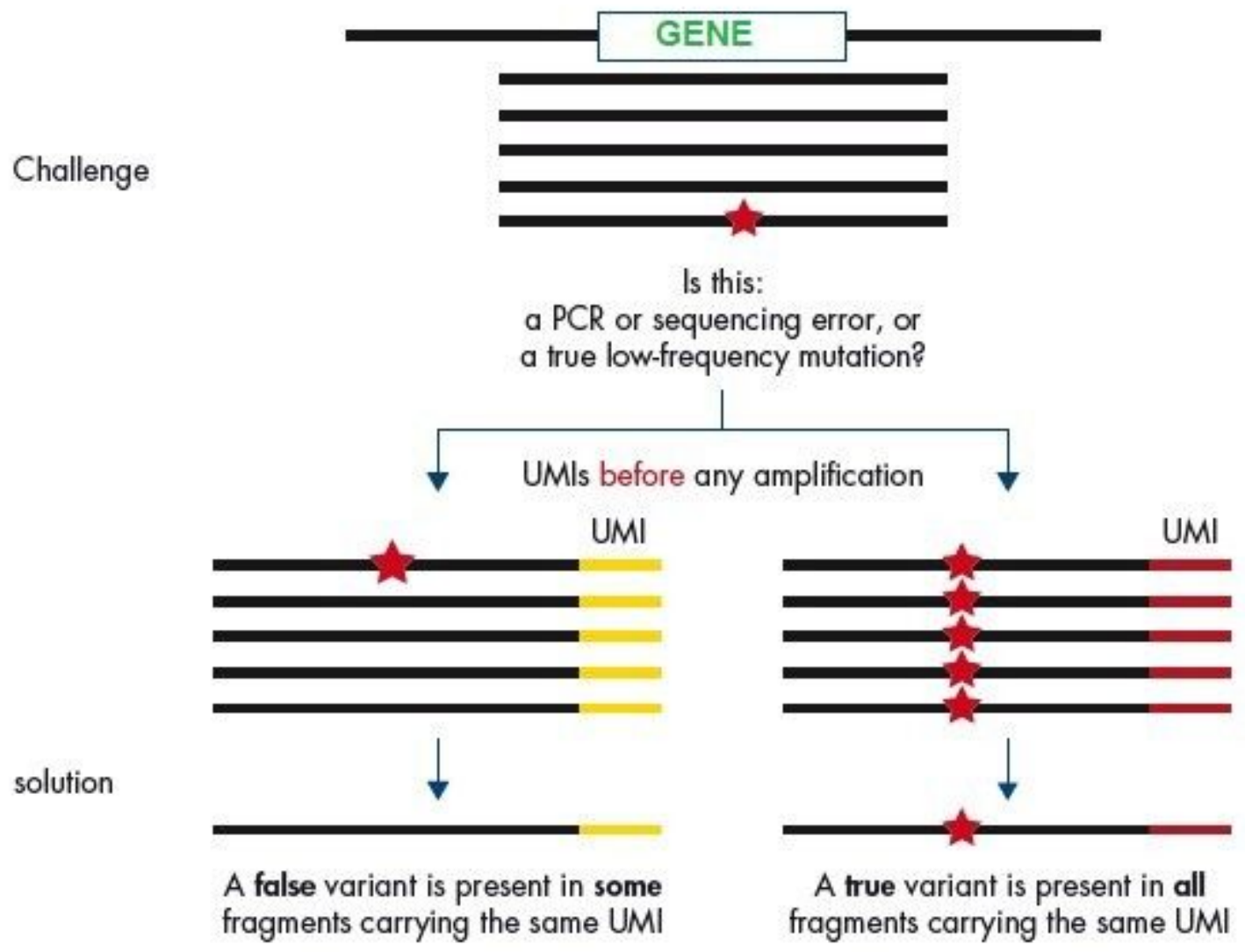

\section{Figure 1}

Mechanism of unique molecular indices (UMIs).

132 newly diagnosed cases

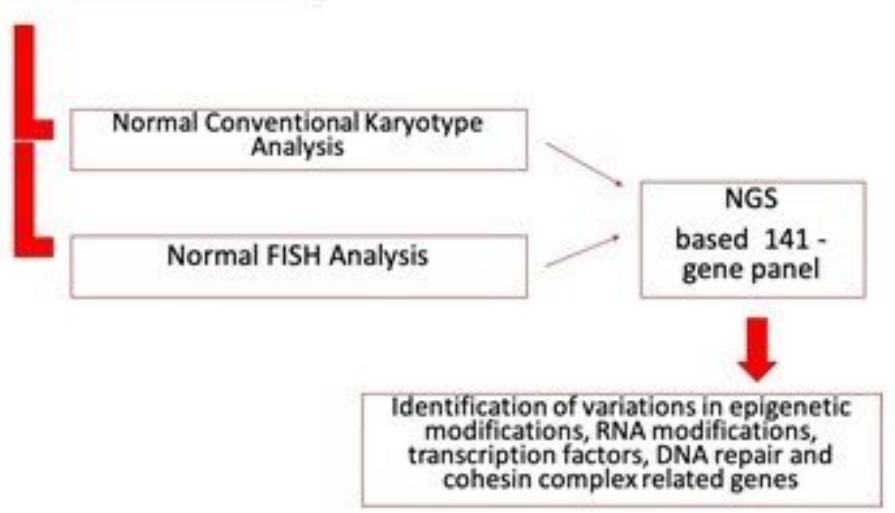

\section{Figure 2}

Suggested algorithm for cytogenetically normal cases. 